\title{
Hybrid mode matching and method of moments method for the full-wave analysis of arbitrarily shaped structures fed through canonical waveguides using only electric currents
}

\author{
Angel Belenguer, Member, IEEE, Héctor Esteban, Member, IEEE, Vicente E. Boria, Senior Member, IEEE, \\ Carmen Bachiller, Member, IEEE, and José V. Morro, Member, IEEE
}

\begin{abstract}
A new hybrid mode matching and method of moments formulation based only on electric currents is presented in this paper. The use of only one equivalent current allows the introduction of a new set of unknowns. The chosen new unknowns are the weights related to the scattered modes that emerge from the ports to the waveguides that feed the problem. Applying this new formulation, the matrices that must be inverted are smaller and the generalized scattering matrix can be obtained directly from the solution of the resulting system of equations, so that no additional projection is needed to obtain the scattering parameters, as happens with traditional approaches with two equivalent currents. As a result, certain efficiency improvement is obtained, as can be seen when this technique is applied to the solution of $\mathbf{H}$ plane problems in rectangular waveguide.
\end{abstract}

Index Terms-Electromagnetic scattering, mode matching methods, moment methods, rectangular waveguides.

\section{INTRODUCTION}

One of the most common techniques employed in developing hybrid formulations is mode matching (MM). MM analyzes discontinuities which separate regions whose modes are known analytically: rectangular waveguides and cavities, circular waveguides and cavities, etc., so that its generality is very limited. However, these structures are very common in microwave devices, and this technique is intensively employed for analyzing a wide range of microwave devices [1]-[4]. MM is very efficient but not general. On the other hand, the general methods, such as the method of moments (MoM), are not as efficient as MM. If we manage to combine MM and MoM we will be able to solve a wide range of problems more efficiently than using MoM alone.

The MM and MoM hybrid formulations are widely used to analyze horns as in [5], where the inner problem is solved using MM and the outer problem is solved by means of MoM. They can also be employed in the solution of resonant cavities of arbitrary geometry when they are fed through several ports [6]-[10]. Traditionally, the problem is discretized by means of

A. Belenguer is with the Departamento de Ingeniería eléctrica, electrónica, automática y comunicaciones, Universidad de Castilla-La Mancha, Escuela Universitaria Politécnica de Cuenca, Campus Universitario, 16071 Cuenca, Spain (e-mail: angel.belenguer@uclm.es).

H. Esteban, V. E. Boria, C. Bachiller, and J. V. Morro are with the Departamento de Comunicaciones, Universidad Politécnica de Valencia, 46022 Valencia, Spain (e-mail: hesteban@dcom.upv.es; vboria@dcom.upv.es; mabacmar@dcom.upv.es; jomorros@dcom.upv.es).
MoM, and then it is solved to find certain equivalent currents, $\vec{J}$ and $\vec{M}$, that model the problem ports. Once the currents have been obtained, the emergent modes from the problem through these ports are computed by means of projecting the current distribution over the modes associated to each port.

The numerical efficiency of these hybrid methods can be improved if we manage to include the weights of the modes in the equations, so the last projection could be avoided.

In order to accomplish this objective, the ports must be represented using only a set of unknowns: $\vec{J}$ or $\vec{M}$. That forces us to eliminate one current in the port characterization, which can be done if the equivalence theorem is applied. Once the port is characterized by means of a single current, it is possible to include the desired weights as unknowns in the system of equations. This has been done in the new technique presented in this paper using only electric currents. The accuracy and efficiency of this new technique has been successfully tested with the analysis of $\mathrm{H}$ plane problems in rectangular waveguides.

\section{EQUiVALENCE}

We need to prove that an arbitrary electromagnetic problem, like the one in the left side of Fig. 1, can be successfully characterized outside $V$ using only electric current densities or only magnetic current densities. This fact has been already used in [11], [12], but we include a proof here to increase the readability of the text.

Applying the equivalence principle, the fields outside an imaginary closed surface, $S$, which in turn encloses certain volume, $V$, can be obtained by placing suitable electric and magnetic current densities over $S$, see Fig. 1 .

The problem is equivalent only outside $S$, because the equivalent sources produce the original fields, $\vec{E}_{2}$ and $\vec{H}_{2}$, only outside $S$. The fields $\vec{E}$ and $\vec{H}$ of Fig. 1 are different from the originals $\vec{E}_{1}$ and $\vec{H}_{1}$ [13].

We can freely choose a solution for the Maxwell equations, $\vec{E}$ and $\vec{H}$ inside $S$, to construct an equivalent problem [13]. From the uniqueness theorem [13] we know that any possible solution of the Maxwell's equations inside $V$ is unambiguously determined by the tangential component of $\vec{E}$, or the tangential component of $\vec{H}$ over $S$.

Then, the first particular solution that we propose, $\vec{E}^{(1)}$ and $\vec{H}^{(1)}$ (see Fig. 2), is the solution of the Maxwell's equations 


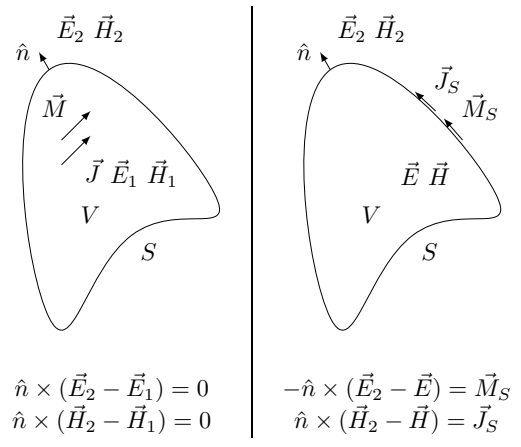

Fig. 1. Equivalence theorem

unambiguously determined by a tangential component of the electric field over $S, \hat{n} \times \vec{E}^{(1)}$, equal to $\hat{n} \times \vec{E}^{(1)}=\hat{n} \times \vec{E}_{2}$. In this case, if we apply the general equivalence principle of Fig. 1, we get these particular equivalent sources, $\vec{M}_{S}^{(1)}=$ $-\hat{n} \times\left(\vec{E}_{2}-\vec{E}^{(1)}\right)=0$ and $\vec{J}_{S}^{(1)}=\hat{n} \times\left(\vec{H}_{2}-\vec{H}^{(1)}\right)$. Therefore, it is possible to find an equivalent problem outside $S$ replacing the original sources by a single electric current density on $S$. In this case, the fields inside $V$ are completely unknown and depend on the fields we are trying to synthesize outside $V$. We only know that these fields exist and could be calculated, but that is enough to conclude that the equivalence is possible.

Traditionally, the Love's equivalence [13] is developed under the assumption that we are not interested in what happens in the unwanted region $(V)$, and maybe this premise could confuse us. Since the Love's equivalence requires two independent sources, one can think that when we are interested in one region we also need two currents to achieve the equivalence. However, in fact, when we apply the Love's equivalence, we are interested in what happens in the unwanted region, unless mathematically speaking, since we force the fields in this region to be zero. That is the reason why the Love's equivalence requires two currents.

Another solution could be the fields, $\vec{E}^{(2)}$ and $\vec{H}^{(2)}$, unambiguously determined by a tangential component of the magnetic field over $S, \hat{n} \times \vec{H}^{(2)}$, equal to $\hat{n} \times \vec{H}^{(2)}=\hat{n} \times \vec{H}_{2}$. Now $\vec{M}_{S}^{(2)}=-\hat{n} \times\left(\vec{E}_{2}-\vec{E}^{(2)}\right)$ and $\vec{J}_{S}^{(2)}=\hat{n} \times\left(\vec{H}_{2}-\vec{H}^{(2)}\right)=0$. Then, it is also possible to find an equivalent problem in terms of a single magnetic current density defined on $S$ (see Fig. 2).

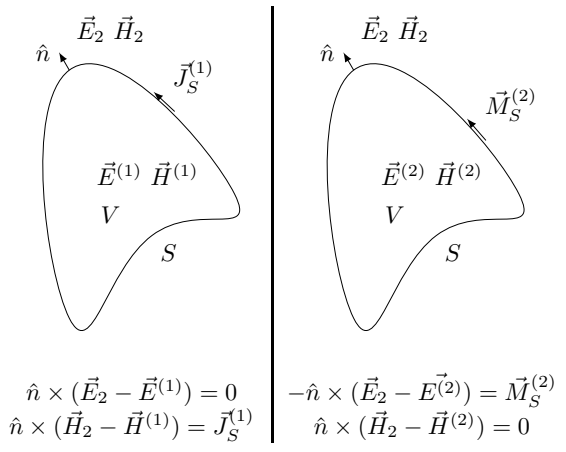

Fig. 2. Equivalences outside $V$ in terms of a single current

Following the same procedure, it is easily shown that an equivalent problem inside $S$ can be expressed in terms of a single electric current density, or in terms of a single magnetic current density, over $S$.

\section{PROBLEM REFORMULATION}

In this section an arbitrarily shaped closed cavity fed through $A$ canonical waveguides is analyzed (see Fig. 3).

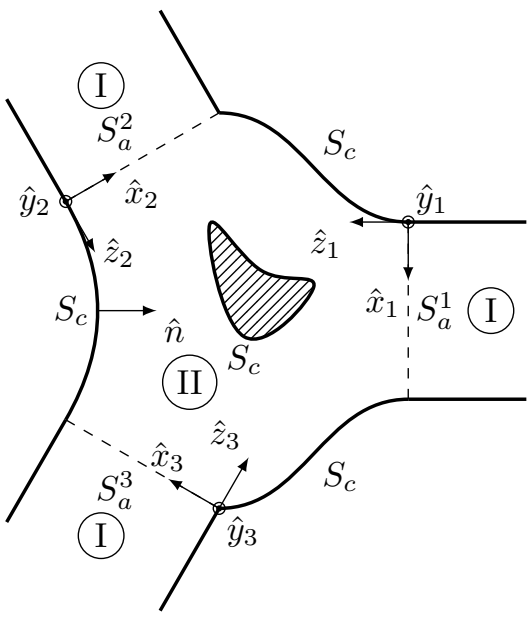

Fig. 3. Example of an arbitrarily shaped closed cavity fed through $A=3$ canonical waveguides

The medium $I I$ is closed, so the equivalence theorem guarantees us that it can be fully characterized by means of a surface electric current density over its limiting surface, that is, $S_{a} \cup S_{c}$, where $S_{a}$ is the surface of all the accessing ports $\left(S_{a}=S_{a}^{1} \cup S_{a}^{2} \cup \cdots \cup S_{a}^{A}\right)$ and $S_{c}$ is the surface of the metallic boundary of the closed cavity (see Fig. 3). That means that the accesses can be fully characterized using a single current, let us say $\vec{J}=\vec{J}_{a}+\vec{J}_{c}$, where $\vec{J}_{a}$ is the surface current defined over $S_{a}$ and $\vec{J}_{c}$ is the surface current defined over $S_{c}$.

In order to obtain $\vec{J}$, we only need to force that the field that this current density produces in the closed medium $I I$ satisfies the corresponding boundary conditions (uniqueness theorem).

The first boundary condition is that the tangential components of the fields have to be continuous over $S_{a}$. The second boundary condition is that the tangential electric field over $S_{c}$ must vanish. Forcing these boundary conditions the following set of equations is obtained

$$
\begin{gathered}
\vec{E}_{i n c, S_{a}^{i}}^{I}+\sum_{n=1}^{N_{i}} b_{n}^{i} \vec{e}_{n, S_{a}^{i}}^{i}=\vec{E}_{S_{a}^{i}}^{I I}\left(\vec{J}_{a}+\vec{J}_{c}\right), \quad i=1,2, \ldots, A \\
\vec{H}_{i n c, S_{a}^{i}}^{I}-\sum_{n=1}^{N_{i}} b_{n}^{i} \vec{h}_{n, S_{a}^{i}}^{i}=\vec{H}_{S_{a}^{i}}^{I I}\left(\vec{J}_{a}+\vec{J}_{c}\right), \quad i=1,2, \ldots, A \\
\vec{E}_{S_{c}}^{I I}\left(\vec{J}_{a}+\vec{J}_{c}\right)=0
\end{gathered}
$$

where

- $b_{n}^{i}$ is the weight of the $n$-th regressive mode in the $i$-th accessing port, and $\vec{e}_{n, S_{a}^{i}}^{i}$ and $\vec{h}_{n, S_{a}^{i}}^{i}$ are the values of the electric and magnetic eigen-vectors for that mode at $S_{a}^{i}$.

- $N_{i}$ is the number of guided modes considered for analysis in the $i$-th port. 
- $\vec{E}_{i n c, S}^{I}$ and $\vec{H}_{i n c, S}^{I}$ are the incident electric and magnetic fields tangential to the surface $S$ in the medium $I$ over any point of $S$.

- $\vec{E}_{S}^{I I}(\vec{J})$ and $\vec{H}_{S}^{I I}(\vec{J})$ are the electric and magnetic fields tangential to the surface $S$ produced by an electric current density $\vec{J}$ in the medium $I I$ over any point of $S$.

In order to simplify the notation it is more convenient to treat all the ports as a single entity. That allows to suppress the index $i$ that refers to the port number in all the port-dependent parameters. Proceeding in this way, we define the following parameters:

$$
\begin{array}{r}
b=\left[b_{1}^{1}, \cdots, b_{N_{1}}^{1}, b_{1}^{2}, \cdots, b_{N_{2}}^{2}, \cdots, b_{1}^{A}, \cdots, b_{N_{A}}^{A}\right] \\
\vec{e}=\left[\vec{e}_{1}^{1}, \cdots, \vec{e}_{N_{1}}^{1}, \vec{e}_{1}^{2}, \cdots, \vec{e}_{N_{2}}^{2}, \cdots, \vec{e}_{1}^{A}, \cdots, \vec{e}_{N_{A}}^{A}\right] \\
\vec{h}=\left[\vec{h}_{1}^{1}, \cdots, \vec{h}_{N_{1}}^{1}, \vec{h}_{1}^{2}, \cdots, \vec{h}_{N_{2}}^{2}, \cdots, \vec{h}_{1}^{A}, \cdots, \vec{h}_{N_{A}}^{A}\right]
\end{array}
$$

where $b, \vec{e}$ and $\vec{h}$ are vectors of $N=\sum_{i=1}^{A} N_{i}$ elements.

To discretize the equations of (1) we will use MoM and we will expand the currents as a weighted sum of certain basis functions

$$
\overrightarrow{J_{c}} \approx \sum_{q=1}^{Q} I_{q} \vec{J}_{q} ; \vec{J}_{a} \approx \sum_{i=1}^{A} \sum_{p=1}^{P_{i}} I_{p i}^{a} \vec{J}_{p i}^{a}=\sum_{p=1}^{P} I_{p}^{a} \vec{J}_{p}^{a}
$$

where $P_{i}$ is the number of basis functions considered for the $i$-th port, $P$ is the number of basis functions for all the accessing ports, and $Q$ the number of basis functions along the conducting surface of the cavity.

The application of MoM is completed by projecting the equations over several sets of test functions.

In order to discretize the last equation of (1) the test functions $\vec{\omega}_{r}(r=1,2, \ldots R, R=Q)$ uniformly placed along $S_{c}$ are used.

The first two equations of (1) force the tangential field continuity over $S_{a}^{i}$. To discretize these equations we project the tangential electric field at the $i$-th port over $S_{i}$ test functions $\left(\vec{u}_{s}^{i}, s=1,2, \ldots S_{i}\right)$ and the tangential magnetic field over $T_{i}$ test functions $\left(\vec{v}_{t}^{i}, t=1,2, \ldots T_{i}\right)$. We have considered $N_{i}$ modes to represent the field in the medium $I$ and we have decomposed the current as a sum of $P_{i}$ basis functions, so we force that $S_{i}=T_{i}=N_{i}=P_{i}$. To sum up, we are considering $S=N$ test functions to evaluate the electric field continuity $\left(\vec{u}_{s}, s=1,2, \ldots S\right)$ and $T=N$ test functions to evaluate the magnetic field continuity $\left(\vec{v}_{t}, t=1,2, \ldots T\right)$ across all ports.

When the equations of (1) are projected over these sets of test functions, a system of equations is obtained. The construction of this matrix system is detailed in the appendix. The solution of this system provides $b_{n}, I_{p}^{a}$ and $I_{q}$.

$$
\left(\begin{array}{ccc}
\underline{\underline{\underline{Z}}}_{11} & \underline{\underline{Z}}_{12} & \underline{\underline{Z}}_{13} \\
\underline{\underline{Z}}_{31}=0 & \underline{\underline{\bar{Z}}}_{32} & \underline{\underline{\underline{Z}}}_{33}
\end{array}\right)\left(\begin{array}{c}
\underline{\underline{b}}^{a} \\
\underline{\underline{I}}^{a} \\
\underline{\underline{I}}^{c}
\end{array}\right)=\left(\begin{array}{c}
\underline{\underline{E}}_{i} \\
\underline{\underline{H}}_{i} \\
0
\end{array}\right)
$$

\section{SCATTERING PARAMETERS}

\section{A. Iterative solution}

The solution of the matrix system of (6) directly provides the amplitudes of all the regressive modes $b_{n}^{i}$. So, in order to compute the $s_{n m}^{i j}$ scattering parameter, we have to excite exclusively the $j$-th port with the $m$-th mode of unitary amplitude, that is, $a_{m j}=1$.

$$
\begin{aligned}
& \vec{E}_{i n c, S_{a}}^{I}= \begin{cases}\vec{e}_{m k}\left(S_{a}^{k}\right) & \text { if } k=j \\
0 & \text { rest }\end{cases} \\
& \vec{H}_{i n c, S_{a}}^{I}= \begin{cases}\vec{h}_{m k}^{I}\left(S_{a}^{k}\right) & \text { if } k=j \\
0 & \text { rest }\end{cases}
\end{aligned}
$$

and with this excitation

$$
s_{n m}^{i j}=b_{n}^{i}, \forall i, n
$$

In addition, an iterative solver [14]-[18] such as biconjugate gradients, biconjugate gradients stabilized, generalized minimal residual, etc., in conjunction with a grouping strategy such as fast multipole method or multilevel fast multipole algorithm [18]-[24] could be used to accelerate the product of

$$
\left(\begin{array}{ll}
\underline{\underline{Z}}_{12} & \underline{\underline{Z}}_{13} \\
\underline{\bar{Z}}_{32} & \underline{\underline{Z}}_{33}^{23}
\end{array}\right)\left(\begin{array}{l}
\underline{\underline{I}}^{a} \\
\underline{\underline{I}}^{c}
\end{array}\right)
$$

The remaining products of (6) are not worth accelerating since $\underline{\underline{Z}}_{11}$ and $\underline{\underline{X}}_{21}$ are block-diagonal and $\underline{\underline{Z}}_{31}$ is equal to zero.

\section{B. Direct solution}

An alternative to using an iterative solver or an acceleration procedure to find the solution of (6) is to obtain the generalized scattering matrix $S$ directly. That can be done if we define

$$
\begin{gathered}
\underline{\underline{Z}}_{a c}=-\underline{\underline{Z}}_{33}^{-1} \underline{\underline{Z}}_{32} \\
\underline{\underline{\underline{X}}}_{G}=\underline{\underline{Z}}_{11}^{-1}\left(\underline{\underline{Z}}_{12}+\underline{\underline{Z}}_{13} \underline{\underline{Z}}_{a c}\right) \\
\left.\underline{\underline{\underline{X}}}_{21}^{-1} \underline{\underline{\underline{X}}}_{22}+\underline{\underline{\underline{X}}}_{23} \underline{\underline{Z}}_{a c}\right)
\end{gathered}
$$

and we take into consideration that $\underline{E}_{i}=-\underline{\underline{Z}}_{11} \underline{a}$ and $\underline{H}_{i}=$ $\underline{\underline{X}}_{21} \underline{a}$. Then the generalized scattering matrix is

$$
\underline{\underline{S}}=-\left\{\underline{\underline{I}}^{+2} \underline{\underline{Z}}_{G}\left[\underline{\underline{X}}_{G}-\underline{\underline{Z}}_{G}\right]^{-1}\right\}
$$

In order to obtain the field inside the medium $I I$ produced in response to any kind of incidence, we could calculate the currents

$$
\begin{gathered}
\underline{I}^{a}=2\left[\underline{\underline{X}}_{G}-\underline{\underline{Z}}_{G}\right]^{-1} \underline{a} \\
\underline{I}^{c}=2 \underline{\underline{Z}}_{a c}\left[\underline{\underline{X}}_{G}-\underline{\underline{Z}}_{G}\right]^{-1} \underline{a}
\end{gathered}
$$

where $\underline{a}$ contains the amplitudes of the incident guided modes. 


\section{EFFICIENCY ANALYSIS}

In order to assess the efficiency of the new technique the number of matrix products (table I) and the number of matrix inversions (table II) required by the new technique are presented in this section and compared with the traditional approach of hybrid MM and MoM formulations with two equivalent currents. It can be observed that the number of operations required to perform the matrix products is the same with the one and two current approaches, but in the case of the matrix inversions the number of operations is smaller with the one current approach.

Moreover, the new technique avoids the projection over the guided modes associated to each port in order to obtain the scattering parameters of the structure, and it also allows the use of an iterative solver and a grouping strategy to accelerate the solution of (6).

TABLE I

MATRIX PRODUCTS AND TOTAL NUMBER OF OPERATIONS REQUIRED

\begin{tabular}{cccc}
\hline & Two currents & \multicolumn{2}{c}{ One current } \\
\hline Prod. & Size & Prod. & Size \\
\hline 1 & $(N+Q) \times(N+Q) \times N$ & 1 & $Q \times Q \times N$ \\
1 & $N \times(N+Q) \times N(N \times N \times N)$ & 2 & $N \times Q \times N$ \\
1 & $N \times N \times N$ & 3 & $N \times N \times N$ \\
\hline Oper. & $Q^{2} N+2 Q N^{2}+3 N^{3}$ & \multicolumn{2}{c}{$Q^{2} N+2 Q N^{2}+3 N^{3}$} \\
\hline
\end{tabular}

TABLE II

MATRIX INVERSIONS AND TOTAL NUMBER OF OPERATIONS REQUIRED

\begin{tabular}{cccc}
\hline & Two currents & \multicolumn{2}{c}{ One current } \\
\hline Inv. & Size & Inv. & Size \\
\hline 1 & $(N+Q) \times(N+Q)$ & 1 & $Q \times Q$ \\
1 & $N \times N$ & 2 & $N \times N$ (bloq. diag.) \\
& & 1 & $N \times N$ \\
\hline Oper. & $O\left(Q^{3}+3 Q N^{2}+3 Q^{2} N+2 N^{3}\right)$ & $O\left(Q^{3}+\left(1+\frac{2}{A^{2}}\right) N^{3}\right)$ \\
\hline
\end{tabular}

\section{PARTicularization FOR H PLANE PROBLEMS IN RECTANGULAR WAVEGUIDE}

\section{A. Election of basis and test functions}

We have applied the general MM and MoM method presented in this paper to the analysis of $\mathrm{H}$ plane problems.

Since $\mathrm{H}$ plane problems in rectangular waveguides are invariant in height, and assuming the incidence of the fundamental $T E_{10}$ mode, only $T E_{m 0}$ modes are needed for a full wave analysis of the structure. As a consequence only the $\hat{y}$ polarization of the electric current is needed (see Figure 3 ).

As basis functions we choose $\vec{J}_{p}^{a}=J_{p}^{a} \hat{y}$ and $\vec{J}_{q}=J_{q} \hat{y}$, where $J_{q}$ and $J_{p}^{a}$ are rectangular pulses uniformly distributed along $S_{c}$ and $S_{a}$.

As test functions we choose $\vec{\omega}_{r}=\omega_{r} \hat{y}$, with $\omega_{r}$ equal to Dirac's delta functions uniformly distributed along $S_{c}$, and $\vec{u}_{s}=u_{s} \hat{y}$ and $\vec{v}_{t}=v_{t} \hat{x}_{i}$, with $u_{s}$ and $v_{t}$ also Dirac's delta functions uniformly distributed along $S_{a}$.

\section{B. Expressions for the fields}

The field expressions of the modes of a rectangular waveguide and the solution of a two-dimensional scattering problem using MoM are very well documented in the literature [25]-[27]. Using those expressions it is quite straightforward to obtain all the expressions needed to apply the new $\operatorname{method}\left(\vec{e}_{m i}^{I}\left(S_{a}^{i}\right), \vec{h}_{m i}^{I}\left(S_{a}^{i}\right), \vec{E}_{S}^{I I}\left(\vec{J}_{p}^{a}\right), \vec{E}_{S}^{I I}\left(\vec{J}_{q}\right), \vec{H}_{S}^{I I}\left(\vec{J}_{p}^{a}\right)\right.$ and $\left.\vec{H}_{S}^{I I}\left(\vec{J}_{q}\right)\right)$.

\section{RESULTS}

In this section we are going to test the accuracy and efficiency of the new method with the analysis of several $\mathrm{H}$ plane problems: a $90^{\circ}$ and a $135^{\circ}$ bend, several beveled $90^{\circ}$ bends and a T-junction with and without load. The geometry and the MoM discretization of all these problems are shown in Figure 4.

In order to test the performance of the new method with complex geometries, the rounded corners that appear due to milling manufacturing have been considered for the loaded T-junction (Figure 4(e)). A radius of $r_{e}=3 \mathrm{~mm}$ has been considered for the rounded corners.

In all the analysis a density of MoM basis functions equal to 100 pulses per wavelength has been used, and in the accessing ports $P_{i}=40$ basis functions provide accurate enough results.

The results of the new method are compared with results from the literature in Figure 5. A very good agreement with results from the literature can be observed.

In Table III the CPU time (Intel Core2Duo with $2.16 \mathrm{GHz}$ clock frequency and $2 \mathrm{~GB}$ of RAM) required by the new method is shown. These results prove the efficiency of the new method both for computing the scattering parameters and for obtaining the field inside the structure.

TABLE III

CPU TIME AND NUMBER OF UNKNOWNS.

\begin{tabular}{cccc}
\hline & Unknowns & $\begin{array}{c}\text { S parameters } \\
\text { sec/freq. } \\
\text { point }\end{array}$ & $\begin{array}{c}E_{y}, H_{x} \text { and } H_{z} \\
\text { sec/freq. point }\end{array}$ \\
\hline $90^{\circ}$ bend & 294 & 0.08 & 5.89 \\
\hline $135^{\circ}$ bend & 182 & 0.03 & 0.71 \\
\hline $\begin{array}{c}\text { Beveled bend } \\
\left(a_{b}=0.98 a\right)\end{array}$ & 260 & 0.06 & 4.23 \\
\hline $\begin{array}{c}\text { Beveled bend } \\
\left(a_{b}=a / \sqrt{2}\right)\end{array}$ & 238 & 0.05 & 2.66 \\
\hline T-junction & 251 & 0.06 & 5.36 \\
\hline Loaded T-junction & 264 & 0.06 & 8.86 \\
\hline $\begin{array}{c}\text { Loaded T-junction } \\
\text { (rounded corners) }\end{array}$ & 329 & 0.10 & 15.78 \\
\hline
\end{tabular}

Figure 6 shows the field pattern inside the T-junction loaded with a cylindrical post and with rounded corners due to milling manufacturing with the incidence of fundamental mode $T E_{10}$.

\section{CONCLUSIONS}

We have developed a new formulation for a hybrid MM and MoM technique that uses only one current, $\vec{J}$. This new formulation can be used to solve arbitrarily shaped problems 


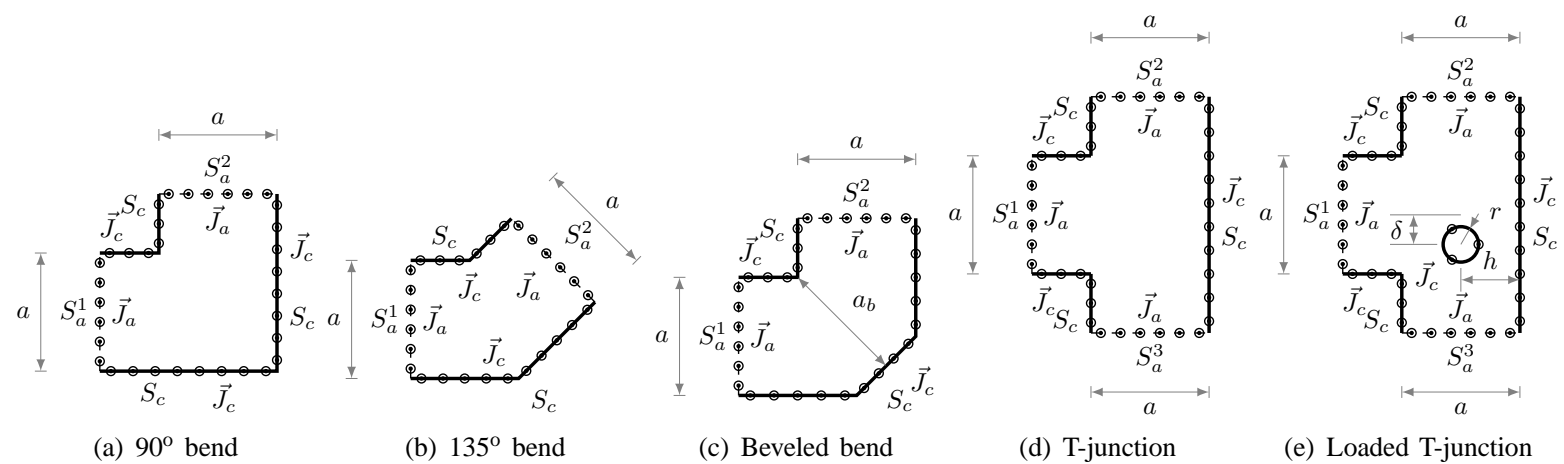

Fig. 4. Geometries and MoM discretization of several $\mathrm{H}$ plane problems. $\delta=0.1 \mathrm{~mm}, h=8,8 \mathrm{~mm}, a=15.799 \mathrm{~mm}$ except for the loaded T-junction $(a=22.86 \mathrm{~mm})$.

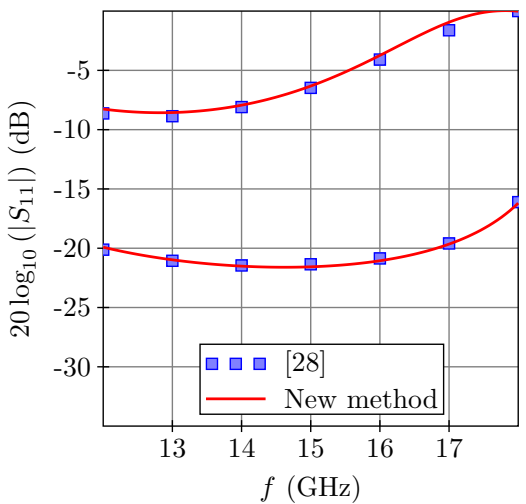

(a) $90^{\circ}$ and $135^{\circ}$ bends

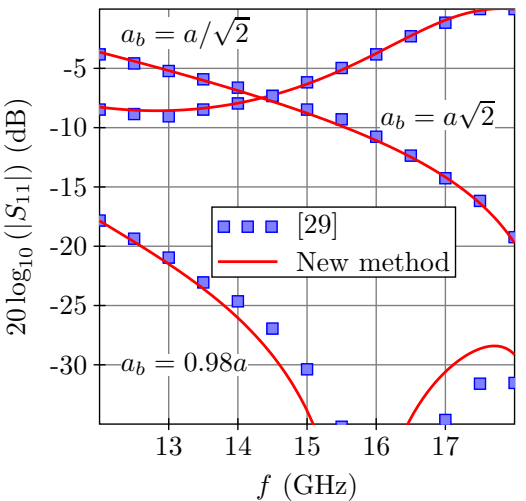

(b) Beveled bends

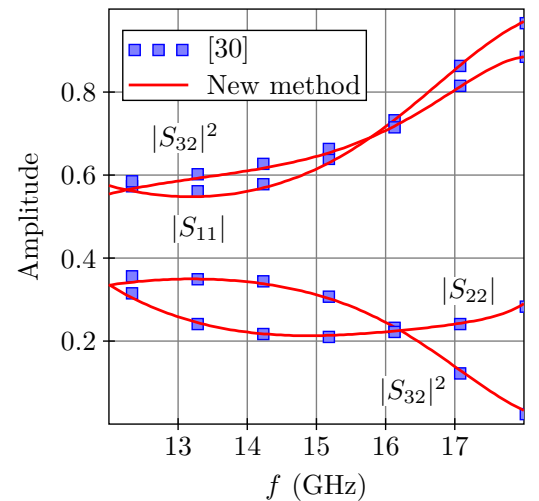

(c) T-junction

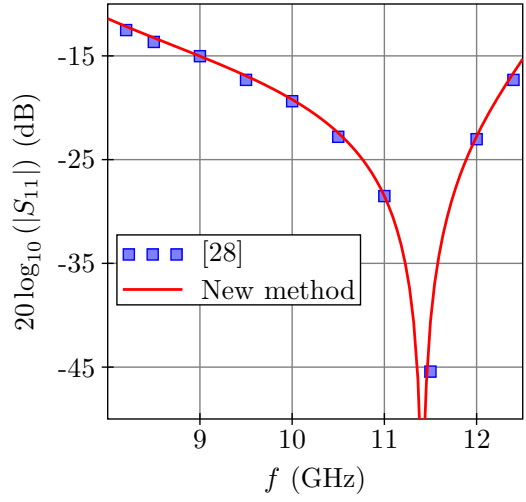

(d) Loaded T-junction

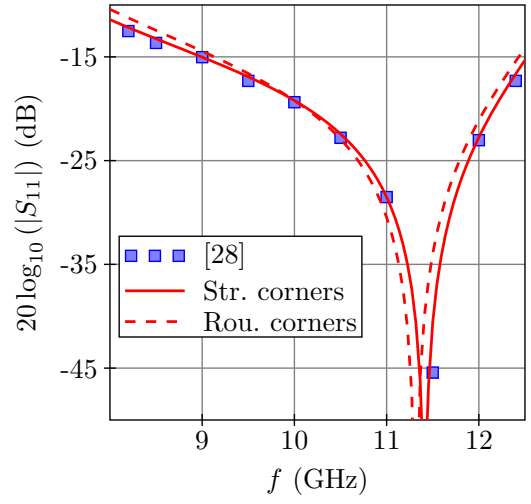

(e) Loaded T-junction $r_{e}=0$ and $r_{e}=3 \mathrm{~mm}$.

Fig. 5. Comparison of the new method and the literature $\left(r_{e}\right.$ is the radius of the rounded corners of the T-junction).

when they are fed through one or more ports using canonical waveguides.

Due to the fact that only one kind of current is needed to characterize the problem ports, we have been able to include, as unknowns of the resulting MoM system of equations, the weights associated to the emerging modes through the ports. Therefore, we are able to compute directly the full generalized scattering matrix of the problem. Then, it is not necessary to project the current over the modes of the feeding waveguides. Moreover, the formulation presented in this paper is suitable for applying iterative solvers such as biconjugate gradients, generalized minimal residual, biconjugate gradients stabilized, etc. and it is also possible to accelerate the solution by means of these iterative solvers, if a grouping strategy like fast multipole method or multilevel fast multipole algorithm is used.

We have successfully applied the new technique to $\mathrm{H}$ plane problems in rectangular waveguides, proving its accuracy and efficiency with these geometries. However, the formulation is fully general and valid for any closed cavity accessed by canonical waveguides. Although we have used only equivalent electric currents in the accessing ports, a similar formulation might have been developed using only equivalent magnetic currents.

\section{ACKNOWLEDGMENT}

This work has been supported by Ministerio de Ciencia e Innovación, Spanish Government, under Research Project 


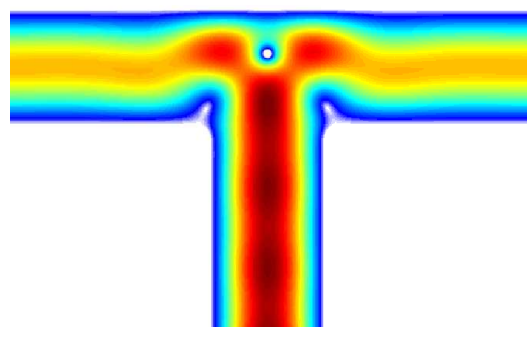

(a) $E_{t}$

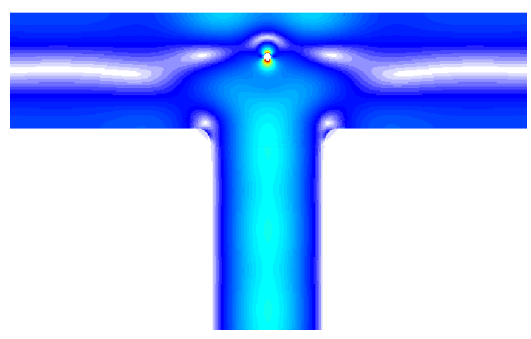

(b) $H_{t}$

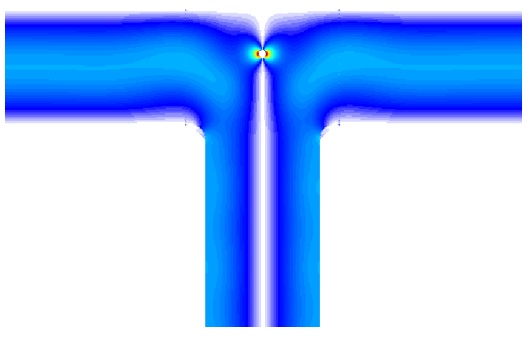

(c) $H_{l}$

Fig. 6. Field pattern inside a loaded T-junction with rounded corners $\left(r_{e}=3 \mathrm{~mm}, a=22.86 \mathrm{~mm}, \delta=0.1 \mathrm{~mm}\right.$ and $\left.h=8,8 \mathrm{~mm}\right)$ with the incidence of the fundamental mode ( $f=11.5 \mathrm{GHz})$ in port 1 . a) Transverse electric field, b) Transversely magnetic field and c) Longitudinal magnetic field.

TEC2007-67630-C03-01.

\section{APPENDIX}

In order to complete the calculation of the system of equations resulting from the discretization of (1), first we need to define the projection operator that will allow us to discretize the equations

$$
<\vec{\kappa}, \vec{v}(x, y, z)>=\iint_{S}[\vec{\kappa} \cdot \vec{v}(x, y, z)] d S
$$

where $\vec{\kappa} \cdot \vec{v}$ is the scalar product between the vectors $\vec{\kappa}$ and $\vec{v}$, $S$ can be $S_{a}$ or $S_{c}$, and $\vec{\kappa}$ is any of the vectorial test functions that we have defined in section III.

Next, we will present the expression for the resulting system of equations after applying a MoM discretization process to (1). In order to do that, first we will organize the unknowns in a column vector $\underline{x}$

$$
\underline{x}^{T}=\left(b_{1}, b_{2}, \cdots, b_{N}, I_{1}^{a}, I_{2}^{a}, \cdots, I_{P}^{a}, I_{1}, I_{2}, \cdots, I_{Q}\right)
$$

Then, we will store the excitation in another column vector, $\underline{c}^{i n c}$ of size $B \times 1, B=S+T+R=N+P+Q$

$$
\underline{c}^{i n c}=\left(\begin{array}{c}
<\tilde{\overrightarrow{\vec{u}}}_{1}, \vec{E}_{i n c, S_{a}}^{I}> \\
<\tilde{\vec{u}}_{2}, \vec{E}_{i n c, S_{a}}^{I}> \\
\vdots \\
<\tilde{\vec{u}}_{S}, \vec{E}_{i n c, S_{a}}^{I}> \\
<\overrightarrow{\vec{v}}_{1}, \vec{H}_{i n c, S_{a}}^{I}> \\
<\tilde{\vec{v}}_{2}, \vec{H}_{i n c, S_{a}}^{I}> \\
\vdots \\
<\tilde{\vec{v}}_{T}, \vec{H}_{i n c, S_{a}}^{I}> \\
0 \\
\vdots \\
0
\end{array}\right\} R
$$

and finally, we will define a $B \times B$ matrix, $\underline{\underline{Z}}$, whose elements can be grouped in the following sub-matrices

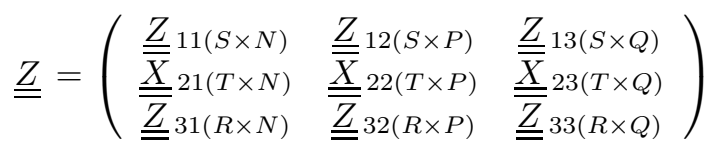

where
- $\underline{\underline{Z}}_{11(S \times N)}$ also can be divided into blocks

$$
\left(\begin{array}{ccc}
\underline{E}_{11\left(S_{1} \times N_{1}\right)} & \cdots & \underline{\underline{E}}_{1 A\left(S_{1} \times N_{A}\right)} \\
\vdots & \ddots & \vdots \\
\underline{\underline{E}} A 1\left(S_{A} \times N_{1}\right) & \cdots & \underline{\underline{E}}_{A A\left(S_{A} \times N_{A}\right)}
\end{array}\right)
$$

and only the diagonal blocks will be non-zero. The elements of a diagonal block, $\underline{\underline{E}} i i$, can be computed

$$
e_{s n}^{(i i)}=-<\vec{u}_{s}, \vec{e}_{n}\left(S_{a}^{i}\right)>
$$

with $s \in$ port $i$ and $n \in$ port $i$.

- $\underline{\underline{Z}}_{12(S \times P)}$

$$
z_{s p}^{(12)}=<\vec{u}_{s}, \vec{E}_{S_{a}^{i}}^{I I}\left(\vec{J}_{p}^{a}\right)>
$$

$s=1,2, \ldots S$ and $p=1,2, \ldots P$.

- $\underline{\underline{Z}}_{13(S \times Q)}$

$$
z_{s q}^{(13)}=<\vec{u}_{s}, \vec{E}_{S_{a}^{i}}^{I I}\left(\vec{J}_{q}\right)>
$$

$s=1,2, \ldots S$ and $q=1,2, \ldots Q$.

- $\underline{\underline{X}}_{21(T \times N)}$, whose elements can also be grouped

$$
\left(\begin{array}{ccc}
\underline{\underline{H}}_{11\left(T_{1} \times N_{1}\right)} & \cdots & \underline{\underline{H}}_{1 A\left(T_{1} \times N_{A}\right)} \\
\vdots & \ddots & \vdots \\
\underline{\underline{H}}_{A 1\left(T_{A} \times N_{1}\right)} & \cdots & \underline{\underline{H}}_{A A\left(T_{A} \times N_{A}\right)}
\end{array}\right)
$$

and a block-diagonal matrix is obtained. The elements of a block in the main diagonal, $\underline{\underline{H}} i i$, can be computed

$$
h_{t n}^{(i i)}=<\vec{v}_{t}, \vec{h}_{n}^{I}\left(S_{a}^{i}\right)>
$$

with $t \in$ port $i$ and $n \in$ port $i$.

- $\underline{\underline{X}}_{22(T \times P)}$

$$
z_{t p}^{(22)}=<\vec{v}_{t}, \vec{H}_{S_{a}^{i}}^{I I}\left(\vec{J}_{p}^{a}\right)>
$$

$t=1,2, \ldots T$ and $p=1,2, \ldots P$.

- $\underline{\underline{X}}_{23(T \times Q)}$

$$
z_{t q}^{(23)}=<\vec{v}_{t}, \vec{H}_{S_{a}^{i}}^{I I}\left(\vec{J}_{q}\right)>
$$

$t=1,2, \ldots T$ and $q=1,2, \ldots Q$.

- $\underline{\underline{Z}}_{31(R \times N)}=0$, because the last equation of (1) does not depend on $b_{k}$.

- $\underline{\underline{Z}}_{32(R \times P)}$

$$
z_{r p}^{(32)}=<\vec{\omega}_{r}, \hat{n}_{S_{c}} \times \vec{E}_{S_{c}}^{T, I I}\left(\vec{J}_{p}^{a}\right)>
$$

$r=1,2, \ldots R$ and $p=1,2, \ldots P$.

- $\underline{\underline{Z}}_{33(R \times Q)}$

$$
z_{r q}^{(33)}=<\vec{\omega}_{r}, \hat{n}_{S_{c}} \times \vec{E}_{S_{c}}^{T, I I}\left(\vec{J}_{q}\right)>
$$


$r=1,2, \ldots R$ and $q=1,2, \ldots Q$.

Finally, a system of equations can be constructed if we form

$$
\underline{\underline{Z}} \underline{x}=\underline{c}^{i n c}
$$

\section{REFERENCES}

[1] A. Wexler, "Solution of waveguide discontinuities by modal analysis," IEEE Trans. Microwave Theory Tech., vol. 15, no. 9, pp. 508-517, September 1967.

[2] S.-N. Reza and R. H. Macphie, "Scattering at rectangular-to-rectangular waveguide junctions," IEEE Trans. Microwave Theory Tech., vol. 30, no. 11 , pp. 2060-2063, 1982

[3] P. Matras, R. Bunger, and F. Amdt, "Mode-matching analysis of the step discontinuity in elliptical waveguides," IEEE Microwave Guided Wave Lett., vol. 6, no. 3, pp. 143-145, March 1996.

[4] O. P. Franza and W. C. Chew, "Recursive mode matching method for multiple waveguide junction modelling," IEEE Trans. Microwave Theory Tech., vol. 44, no. 1, pp. 87-92, January 1996.

[5] R. Bunger, R. Beyer, and F. Arndt, "Rigorous combined Mode-Matching integral equation analysis of horn antennas with arbitrary cross section," IEEE Trans. Antennas Propagat., vol. 47, no. 11, pp. 1641-1648, November 1999.

[6] R. Bunger and F. Arndt, "Moment-Method analysis of arbitrary 3-D metallic N-port waveguide structures," IEEE Trans. Microwave Theory Tech., vol. 48, no. 4, pp. 531-537, April 2000.

[7] F. Arndt, V. Catina, and J. Brandt, "Flexible hybrid MM/MoM technique for the CAD and optimization of arbitrarily shaped 3D waveguide components," in Proc. of the 33rd European Microwave Conference, Munich, Germany, October 2003, pp. 343-346.

[8] F. Arndt, J. Brandt, V. Catina, J. Ritter, I. Rullhusen, J. Dauelsberg, U. Hilgefort, and W. Wessel, "Fast CAD and optimization of waveguide components and aperture antennas by hybrid MM/FE/MoM/FD methods state-of-the-art and recent advances," IEEE Trans. Microwave Theory Tech., vol. 52, no. 1, pp. 292-305, January 2004.

[9] V. Catina, F. Arndt, and J. Brandt, "A surface integral equation formulation for dielectric post structures in waveguides," in Microwave Symposium Digest, 2005 IEEE MTT-S International, June 2005, pp. 4 pp.-.

[10] $\stackrel{\text { pp.-. }}{\longrightarrow}$ "Hybrid surface integral-equation/mode-matching method for the analysis of dielectric loaded waveguide filters of arbitrary shape," IEEE Trans. Microwave Theory Tech., vol. 53, no. 11, pp. 3562-3567, November 2005.

[11] A. W. Glisson, "An integral equation for electromagnetic scattering from homogeneous dielectric bodies," IEEE Trans. Antennas Propagat., vol. 32, no. 2, pp. 173-175, February 1984.

[12] M. S. Yeung, "Single integral equation for electromagnetic scattering by three-dimensional homogeneous dielectric objects," IEEE Trans. Antennas Propagat., vol. 47, no. 10, pp. 1615-1622, October 1999.

[13] C. A. Balanis, Antenna theory : analysis and design, 2nd ed. New York: John Wiley \& Sons, Inc., 1997.

[14] R. Barrett, M. Berry, T. F. Chan, J. Demmel, J. Donato, J. Dongarra, V. Eijkhout, R. Pozo, C. Romine, and H. V. der Vorst, Templates for the Solution of Linear Systems: Building Blocks for Iterative Methods, 2nd ed. Philadelphia, PA: SIAM, 1994.

[15] Y. Saad, Iterative Methods for Sparse Linear Systems, 2nd ed. SIAM, January 2003.

[16] J. Lee, J. Zhang, and C.-C. Lu, "Performance of preconditioned Krylov iterative methods for solving hybrid integral equations in electromagnetics," Journal of Applied Computational Electromagnetics Society, vol. 18, no. 3, pp. 54-61, 2003.

[17] A. F. Peterson and R. Mittra, "Convergence of the Conjugate Gradients Method when applied to matrix equations representing electromagnetic scattering problems," IEEE Trans. Antennas Propagat., vol. 34, no. 12, pp. 1447-1454, December 1986.

[18] J. Song and W. C. Chew, "Fast Multipole Method solution of combined field integral equation," in 11th Annual Review of Progress in Applied Computational Electromagnetics, vol. 1. Monterey, California: Applied Computational Electromagnetics Society, March 1995, pp. 629-636.

[19] V. Rokhlin, "Rapid solution of integral equations of scattering theory in two dimensions," Journal of Computational Physics, vol. 86, no. 2, pp. 414-439, February 1990.

[20] N. Engheta, W. D. Murphy, V. Rokhlin, and M. Vassiliou, "The Fast Multipole Method (FMM) for electromagnetic scattering problems," IEEE Trans. Antennas Propagat., vol. 40, no. 6, pp. 634-641, June 1992.
[21] R. Coifman, V. Rokhlin, and S. Wandzura, "The Fast Multipole Method for the wave equation: A pedestrian prescription," IEEE Antennas Propagat. Magazine, vol. 35, no. 3, pp. 7-12, February 1993.

[22] J. Song, C.-C. Lu, and W. C. Chew, "Multilevel Fast Multipole Algorithm for electromagnetic scattering by large complex objects," IEEE Trans. Antennas Propagat., vol. 45, no. 10, pp. 1488-1493, October 1997.

[23] J. Song and W. C. Chew, "Multilevel Fast Multipole Algorithm for solving combined field integral equation of electromagnetic scattering," Microwave and Optical Technology Letters, vol. 10, no. 1, pp. 14-19, September 1995.

[24] X. Q. Sheng, J.-M. Jin, J. Song, W. C. Chew, and C.-C. Lu, "Solution of combined-field integral equation using Multilevel Fast Multipole Algorithm for scattering by homogeneous bodies," IEEE Trans. Antennas Propagat., vol. 46, no. 11, pp. 1718-1726, November 1998.

[25] N. Marcuvitz, Waveguide Handbook. London: IEE Electromagnetic Wave Series 21, 1986.

[26] R. F. Harrington, Field Computation by Moment Methods, ser. IEEE Press / OUP Series on Electromagnetic Wave Theory. IEEE Press / OUP, 1993.

[27] A. F. Peterson, S. L. Ray, and R. Mittra, Computational Methods for Electromagnetics, ser. IEEE Press / OUP Series on Electromagnetic Wave Theory. IEEE Press / OUP, 1997.

[28] J. Reiter and F. Arndt, "Rigorous analysis of arbitrarily shaped H- and Eplane discontinuities in rectangular waveguides by a full-wave boundary contour mode-matching method," IEEE Trans. Microwave Theory Tech., vol. 43, no. 4, pp. 796-801, April 1995.

[29] _ _ "A full-wave boundary contour mode-matching method BCMM for the rigorous CAD of single and cascaded optimized H-plane and E-plane bends," Microwave Symposium Digest, 1994., IEEE MTT-S International, pp. 1021-1024, May 1994.

[30] X.-P. Liang, K. Zaki, and A. Atia, "A rigorous three plane modematching technique for characterizing waveguide t-junctions, and its application in multiplexer design," IEEE Trans. Microwave Theory Tech., vol. 39, no. 12, pp. 2138-2147, December 1991.

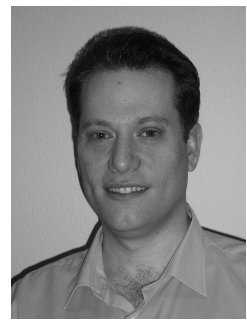

Ángel Belenguer Martínez received his degree in telecommunications engineering from the Universidad Politécnica de Valencia (UPV), Spain, in 2000, and his Ph.D. degree, also from the UPV, in 2009. He joined the Universidad de Castilla-La Mancha in 2000, where he is now Profesor Titular de Escuela Universitaria in the Departamento de Ingenieria Electrica, Electronica, Automatica y Comunicaciones. His research interests include methods in the frequency domain for the full-wave analysis of open-space and guided multiple scattering problems, specifically the improvement or acceleration of these methods using several strategies: hybridization of two or more different methods, the use of specific basis and/or particular Green functions, and the application of accelerated solvers or solving strategies (like grouping) to new problems or structures.

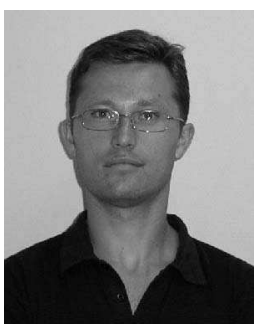

Héctor Esteban González received a degree in telecommunications engineering from the Universidad Politécnica de Valencia (UPV), Spain, in 1996, and a Ph.D. degree in 2002. He worked with the Joint Research Centre, European Commission, Ispra, Italy. In 1997, he was with the European Topic Centre on Soil (European Environment Agency). He rejoined the UPV in 1998. His research interests include methods for the full- wave analysis of open-space and guided multiple scattering problems, CAD design of microwave devices, electromagnetic characterization of dielectric and magnetic bodies, and the acceleration of electromagnetic analysis methods using the wavelets and the fast multipole method. 


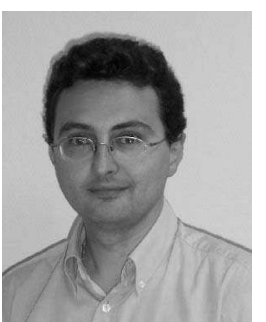

Vicente E. Boria Esbert received the Ingeniero de Telecomunicación and the Doctor Ingeniero de Telecomunicación degrees from the Universidad Politécnica de Valencia, Spain, in 1993 and 1997. In 1993 he joined the Universidad Politécnica de Valencia where he is Full Professor since 2003. In 1995 and 1996 he was held a Spanish Trainee position with the European Space research and Technology Centre (ESTEC)-European Space Agency (ESA). He has served on the Editorial Boards of the IEEE Transactions on Microwave Theory and Techniques. His current research interests include numerical methods for the analysis of waveguide and scattering structures, automated design of waveguide components, radiating systems, measurement techniques, and power effects in passive waveguide systems.

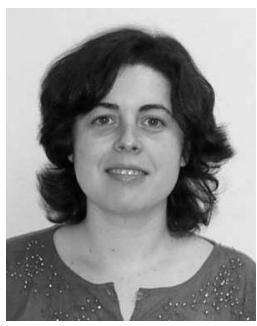

Carmen Bachiller received her degree in communication engineering from the Universidad Politécnica de Valencia in 1996. She worked from 1997 to 2001 in the ETRA I+D company as a project engineer in research and development on automatic traffic control, public transport management and public information systems using telecommunication technology. In 2001 she joined the Communication Department of the Universidad Politécnica de Valencia as an assistant lecturer. She is teaching signal and systems theory and microwaves. She has participated in several teaching innovation projects. She is now working in her Ph.D. thesis on electromagnetism and radio frequency circuits.

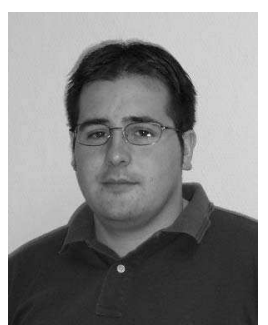

José Vicente Morro received the telecommunications engineering degree from the Universidad Politécnica de Valencia (UPV), Valencia, Spain, in 2001 , and is currently pursuing his Ph.D. degree at the UPV. In 2001, he became a Research Fellow with the Departamento de Comunicaciones, UPV. In 2003 he joined the Signal Theory and Communications Division, Universidad Miguel Hernández, where he was a Lecturer. In 2005, he rejoined the Departamento de Comunicaciones, UPV, as an Assistant Lecturer. His current interests include CAD design of microwave devices and EM optimization methods. 